\title{
Influence de la durée de la restriction hydrique sur la production de la lime Tahiti (Citrus aurantifolia Christm. Swing.) en Guinée
}

Mamadou Tidiane Sankaréla Diallo*, Gnagna Gboulomou

Irag BP 1523,

Conakry, Guinée

ou

CRA Foulaya BP 156,

Kindia, Guinée

\author{
Effects of water restriction on Tahiti lime tree (Citrus aurantifolia Christm. \\ Swing.) yields in Guinea.
}

Abstract - Introduction. In Guinea, citrus cultivation is subject to the return of the rainy season. Tree production is grouped from October to January and, the rest of the year, citrus fruit is almost nonexistent on the market. A step, aiming to shift the production of the citrus trees, and of the Tahiti lime tree in particular, was undertaken by better control of the duration of the water restriction period. Materials and methods. The study, carried out from 1996 to 1999 at the Foulaya site in Guinea, made it possible to compare six treatments, among which were a treatment of trees with continuous irrigation and five treatments with various durations of water restrictions [total dry season or drought of $(30,45,60$ and 75) d]. The experimental design consisted of four random blocks and the elementary plot of two trees per treatment. The flowering and the production of the treated trees were studied during four harvest campaigns. Results and discussion. Various observations were made concerning the recovery of the growth, the flowering date, density and duration, and the production. The continuous irrigation had a depressive effect on the yield of $-12,6 \%$. Production was extended to ( 4 to 5 ) additional months (the end of April to August) allowing a longer availability of the Tahiti lime in the market. The value increase of out of season production was 1.5 to 2.5 times as much as that obtained in the normal season. Conclusion. Drought periods limited to (30 or 45) d by controlling the irrigation made it possible to shift and obtain economically profitable annual average yields of (16.8 and 15.1) th ha ${ }^{-1}$ of Tahiti limes. This approach could be reproduced advantageously in commercial plantations of Tahiti lime trees for out of season harvests.

Guinea / Citrus aurantifolia / drought stress / out of season products / flowering / yields / irrigation scheduling

\section{Influence de la durée de la restriction hydrique sur la production de la lime Tahiti (Citrus aurantifolia Christm. Swing.) en Guinée.}

Résumé - Introduction. En Guinée, la culture des agrumes est soumise au retour de la saison pluvieuse. Il s'ensuit que leur production est groupée d'octobre à janvier et que, le reste de l'année, ces fruits sont quasiment inexistants sur le marché. Une démarche visant à décaler la production de ces arbres, et du limettier Tahiti en particulier, a été entreprise à partir d'un meilleur contrôle de la durée de la période de sécheresse. Matériel et méthodes. L'étude conduite de 1996 à 1999 dans la station de Foulaya en Guinée a permis de comparer six traitements constitués d'un traitement d'arbres en irrigation continue et de cinq traitements de durées de restriction hydrique variables [saison sèche totale ou sécheresse de $(30,45,60$ et 75) j]. Le dispositif a été constitué de quatre blocs aléatoires et la parcelle élémentaire de deux arbres par traitement. La floraison et la production des arbres traités ont été étudiées pendant quatre campagnes de récolte. Résultats et discussion. Des réponses variées ont été observées sur la reprise de la croissance, la date, les densité et durée de la floraison et la production. L'irrigation continue a eu un effet dépressif sur le rendement de $-12,6 \%$. La production a été prolongée sur ( 4 à 5) mois supplémentaires (fin avril à août) permettant une disponibilité plus longue de la lime Tahiti sur le marché. La plus-value de la production de contresaison a été de 1,5 à 2,5 fois celle obtenue en saison normale. Conclusion. Des périodes de sécheresse limitées à (30 ou 45) j par le contrôle de l'irrigation ont permis de décaler et d'obtenir des productions moyennes annuelles de $(16,8$ et 15,1$) \mathrm{t} \cdot \mathrm{ha}^{-1}$ de limes économiquement plus rentables. Cette approche pourrait être reproduite avantageusement en plantations commerciales de limes Tahiti de contre-saison.

Fruits, 2002, vol. 57, p. 27 (C) 2002 Cirad/EDP Sciences All rights reserved DOI: 10.1051/fruits:2002024

REsumen EsPañol, p. 285
Guinée / Citrus aurantifolia / stress dû à la sécheresse / culture de contre-saison / floraison / rendement / calendrier des irrigations 


\section{Introduction}

Avec une production annuelle de l'ordre de (50 000 à 60 000) t, les agrumes constituent, en Guinée, l'une des principale cultures fruitières d'importance économique. Il existe dans ce pays une vocation et une tradition pour cette culture, soutenues par des conditions écologiques généralement favorables et par des débouchés certains tant à l'échelle nationale que régionale (Sénégal, Gambie, Guinée Bissau).

Dans les vergers de case et les nouvelles plantations, les mandariniers et les limettiers ont tendance à s'implanter de plus en plus auprès des traditionnels orangers dont ils complètent la production tout en la concurrençant. La demande de limes, en particulier, est croissante d'année en année pendant le carême, la saison sèche ou lors de cérémonies festives. Le prix du kg de limes et de citrons, qui oscille en moyenne entre (250 à 750) francs guinéens ${ }^{1}$ (FG) sur les marchés urbains, est fort attractif. À Boké, dans les zones industrielles de Kamsar et Sangarédi, le jus naturel de limes et de citrons est vendu $1000 \mathrm{FG} \cdot \mathrm{kg}^{-1}$.

Parmi les espèces d'agrumes exploitées en Guinée, la lime Tahiti (Citrus aurantifolia Christm. Swing. ou Citrus latifolia Tan.) se distingue en particulier par une bonne réponse à l'irrigation et à la fertilisation, une croissance active, une floraison remontante, une mise à fruits précoce et une production régulière. Sur des arbres âgés de (3 à 6) ans, une moyenne de (25 à 50) kg de fruits par arbre et par an [1] peuvent être récoltés.

Considérée en outre comme une plante indicatrice des conditions de vie du verger, la lime Tahiti exprime de manière précise les manifestations ou symptômes d'excès et/ou insuffisances hydriques et nutritionnelles de son environnement.

\subsection{Besoins en eau et stress hydriques}

De nombreuses espèces fruitières cultivées en zones tropicales et méditerranéennes ont des besoins importants en eau. Dans la majorité des cas, les agronomes ou les agriculteurs concernés par la gestion des exploitations fruitières sont donc confrontés au problème d'optimisation de l'alimentation hydrique des plantes.

Trois approches sont couramment utilisées pour évaluer les besoins en eau d'une culture :

- mesure de l'évapotranspiration potentielle (ETP) et relative (ETR) à partir de certaines données climatiques ;

- évaluation du stock d'eau disponible, du potentiel hydrique, de la conductivité hydraulique et du bilan hydrique dans le sol ;

- mesures effectuées sur la plante ellemême : micrométrie des organes, mesure des flux de sève, radiothermométrie ou potentiel foliaire.

Une autre approche a été moins explorée. Il s'agit de la gestion de l'alimentation hydrique des plantes par la maîtrise de la durée de privation d'eau. Cela se traduit par la détermination du temps de sécheresse minimal à respecter avant de recourir à l'irrigation d'un verger. Cette technique a l'avantage d'être simple, peu onéreuse et facilement mise en ouvre.

Dans les régions tropicales, la production d'agrumes, et donc de limes, est soumise au rétablissement des précipitations naturelles et à leur durée [2, 3]. Il s'ensuit deux périodes de floraison qui n'ont pas la même intensité, la plus importante ayant lieu aux mois de mars/avril, et la plus faible en septembre/octobre [2-4].

Plus précisément, dans les conditions guinéennes, la saison pluvieuse s'étale de mai à octobre. Le retour des pluies, uniforme sur l'ensemble des zones productrices d'agrumes, induit une floraison groupée dans les vergers et donc une concentration de la production de limes, de citrons et d'oranges entre la mi-septembre et la fin décembre, voire le mois de janvier. Les limettiers produisent d'abord, puis les citronniers, puis les orangers. De novembre à avril, la saison sèche s'installe entraînant un déficit hydrique qui s'intensifie dans le sol et les plantes. Les arbres entrent dans un état de flétrissement qui va en s'intensifiant et il se produit, alors, au sein du 
verger, des perturbations physiologiques et métaboliques dont les conséquences sont souvent irréversibles : échec de la floraison ou de la nouaison, chute de fruits en croissance, dessèchement de rameaux et mort de certains plants [5-8].

La restriction hydrique provoquée par la fin de la saison pluvieuse et le début de la saison sèche est l'un des facteurs limitants de la culture des agrumes. Dans les vergers non irrigués, il n'est pas possible d'évaluer les performances maximales potentielles des variétés et des porte-greffes ; de même, les effets d'apports d'éléments fertilisants N-P-K sont difficiles à évaluer [9, 10]. En revanche, l'utilisation de parcelles irriguées permet de maîtriser la durée de la période de sécheresse et d'envisager la production étalée et rentable de limes de contre-saison. Cette technique serait la plus performante pour améliorer la croissance, la productivité et l'étalement des récoltes [9].

Les besoins en eau des limes sont importants et varient de (1 000 à 1 500) $\mathrm{mm} \cdot \mathrm{ha}^{-1} \cdot \mathrm{an}^{-1}$. En saison sèche, ils ne peuvent être satisfaits que par des arrosages permettant d'apporter de l'eau aux arbres, au moment et aux quantités qu'ils requièrent. Ces deux éléments complémentaires - fréquences et doses d'irrigation -, susceptibles d'évoluer en sens inverse selon la nature du sol, sont cependant déterminants dans un essai d'étude des besoins en eau de la culture [5, 7, 9].

Toutefois, des travaux effectués sur différentes espèces fruitières (agrumes, manguiers, pêchers, etc.) ont montré l'effet bénéfique d'un stress hydrique sur l'intensification de la floraison lorsque l'alimentation en eau redevient adéquate. Les stress hydriques peuvent donc, sous certaines conditions, favoriser à la fois l'augmentation et l'étalement de la production [5, 7, 9, 11-13].

La conduite optimisée de l'irrigation des arbres fruitiers nécessite à la fois la maîtrise des apports pendant les périodes d'irrigation et la connaissance des effets de la restriction de l'alimentation hydrique [14].

\subsection{Réponse du limettier au stress hydrique}

Dès 1866, une production de contre-saison du citronnier avait pu être obtenue en Italie par forçage des arbres. Cette technique a été reprise ensuite dans d'autres régions productrices d'agrumes présentant des conditions climatiques diverses : USA (Californie), Guinée, Antilles, Cameroun, Madagascar, etc. [2, 3, 10]. Ces expérimentations ont montré qu'il était possible de faire fleurir les agrumes après une période de stress hydrique $[3,9,10]$. Néanmoins, du fait que de nombreux autres facteurs, tels que la durée d' ensoleillement, les périodes de floraison et de nouaison, etc., interfèrent sur le phénomène, aucune corrélation directe n'a pu être établie entre la durée de la restriction hydrique et l'intensité de la production obtenue.

La réponse des agrumes à l'interruption de la période de sécheresse se traduit par l'épanouissement des fleurs ; cet évènement a lieu entre (20 et 28) $\mathrm{j}$ après la reprise de l'apport d'eau aux plants selon certains chercheurs $[1,3,6]$ et entre la quatrième et cinquième semaine selon d'autres [2, 7]. En Sicile et en Californie, il a été possible d'induire le déclenchement de la floraison des limettiers en interrompant la période de sécheresse estivale [7]. Des observations similaires ont été rapportées sur pêcher par Ali et al. [13] et Chalmers et al. [14]. Globalement l'interruption de la restriction hydrique se traduirait par la formation de rameaux végétatifs, florifères et mixtes [12, 13, 15]. Cependant, bien que le nombre de boutons floraux et de fleurs individuelles par branche augmente, la croissance des racines, la synthèse d'auxine et le transport de la cytokine des racines en direction de la frondaison semblent ralentir [7].

Les limettiers mieux adaptés au climat tropical que les orangers et, par ailleurs, susceptibles de présenter une floraison remontante répondent mieux au stress hydrique [10, 11, 15].

En Guinée, les recherches effectuées entre 1953 et 1957 dans des plantations d'agrumes âgées de (5 à 7) ans ont permis de montrer qu'il était possible de contrôler 
la date de floraison par la mise en route des premières irrigations qui mettent fin à la restriction hydrique infligée par la saison sèche.

À partir de ces résultats, il est apparu utile de mener une étude expérimentale, sur le limettier de Tahiti, permettant de tester plusieurs durées de sécheresse, dans le contexte des conditions climatiques actuelles du pays.

Outre l'effet sur l'intensité de floraison, un décalage des dates de production des agrumes pourrait permettre un meilleur contrôle de la cercosporiose ou phaeoramulariose des agrumes, maladie causée par Phaeoramularia angolensis et apparue en 1993 dans les zones montagneuses et humides du pays (moyenne Guinée et Guinée forestière) ; en effet, les floraisons de saisons sèches pourraient être moins vulnérables vis-à-vis de cette maladie que ne le sont celles de périodes pluvieuses.

Les travaux présentés ont cherché à étudier l'influence de la durée de la restriction hydrique, qui peut entraîner un stress hydrique, sur la production du limettier Tahiti. De ce fait, l'incidence de cette privation d'eau sur les variations de l'état hydrique des arbres [16] et du sol [17, 18] n'a pas été évaluée et donc les potentiels hydriques n'ont pas été calculés.

La détermination de la durée de restriction hydrique, la plus appropriée dans la production de limes Tahiti de contre- saison, pourra être exploitée pour établir conjointement une tendance pour la production décalée d'oranges des zones envahies par la cescosporiose.

\section{Matériel et méthodes}

\subsection{Site expérimental}

L'essai a été conduit entre octobre 1996 et juillet 1999 à Méhenyi, dans l'un des vergers de la station de Foulaya (Guinée) située à $10^{\circ} 3^{\prime}$ de latitude $\mathrm{N}, 12^{\circ} 52^{\prime}$ de longitude $\mathrm{O}$ et $380 \mathrm{~m}$ d'altitude. Le sol de ce verger est de type ferralitique profond à texture sablo-limono-argileuse et convient bien à la culture des agrumes ; le pH est moyennement acide $(\mathrm{pH}=4,8)$.

Afin de pouvoir évaluer la durée de la privation d'eau et l'influence éventuelle de certains paramètres agro-météorologiques pouvant interférer avec la production, les dates de début et de fin de la grande saison des pluies (tableau I), les températures, humidités relatives et composantes de la pluviométrie ont été relevées (figure 1).

\subsection{Matériel végétal}

L'étude a porté sur des limettiers (Citrus aurantifolia Christm. Swingle ou Citrus latifolia Tanaka) de la variété Tahiti, plantés en 1990 à $(9 \times 7) \mathrm{m}$, soit à une densité de 158 plants $\cdot$ ha $^{-1}$.

\begin{tabular}{|c|c|c|c|c|}
\hline \multirow{2}{*}{$\begin{array}{l}\text { Année } \\
\text { d'observation }\end{array}$} & \multicolumn{2}{|c|}{ Reprise des pluies } & \multicolumn{2}{|c|}{ Dernières pluies } \\
\hline & Date & $\begin{array}{l}\text { Quantité } \\
\text { (mm) }\end{array}$ & Date & $\begin{array}{l}\text { Quantité } \\
\text { (mm) }\end{array}$ \\
\hline 1996 & 23 avril & 67,0 & 31 octobre & 0,6 \\
\hline 1997 & 11 avril & 50,4 & 19 novembre & 2,8 \\
\hline 1998 & 19 mai & 47,8 & 26 octobre & 6,7 \\
\hline 1999 & 20 mai & 43,9 & - & - \\
\hline
\end{tabular}


Le verger a été entretenu par desherbages manuels et chimiques périodiques ; la fertilisation a été fractionnée et des traitements phytosanitaires spécifiques ont été appliqués contre les termites, les pucerons et les maladies fongiques.

\subsection{Préparation de la parcelle}

Afin de pouvoir contrôler l'irrigation du verger durant la saison sèche, la parcelle expérimentale a été desherbée chaque année en octobre, donc à la fin de la saison pluvieuse, et un réseau de rigoles et de doubles cuvettes a été mis en place. Les rigoles - une par double lignes d'arbres - ont été connectées par simples débouchages et rebouchages à la terre, à un canal principal alimenté en eau. Des rigoles latérales ont permis de relier les rigoles principales à des cuvettes aménagées au pied des arbres, qui ont pu être ainsi remplies au moment voulu et à la dose désirée.

La surface des cuvettes a varié de (9 à 14) $\mathrm{m}^{2}$ suivant l'âge et le développement de la frondaison des arbres. Le volume d'eau d'irrigation apporté a été déterminé à l'aide d'une formule donnée par la littérature : $\mathrm{Q}=\mathrm{S}_{\mathrm{c}} \times \mathrm{P}_{\mathrm{u}} \times \mathrm{C}_{\mathrm{r}} \times \mathrm{R}_{\mathrm{fu}}$, où $\mathrm{S}_{\mathrm{c}}$ est la surface de la cuvette ; $\mathrm{P}_{\mathrm{u}}$, la profondeur utile des racines $(0,5 \mathrm{~m}) ; \mathrm{C}_{\mathrm{r}}$, la capacité de rétention $(7,5 \%) ; \mathrm{R}_{\mathrm{fu}}$, la réserve d'eau facilement utilisable en \% (45\%). Le calcul, basé donc sur la surface de la cuvette, a permis un apport d'eau de $1000 \mathrm{~L} \cdot \mathrm{mois}^{-1}$ (soit $100 \mathrm{~mm} \cdot \mathrm{mois}^{-1}$ ).

\subsection{Dispositif expérimental et traitements}

Six traitements basés sur un contrôle de la durée de restriction hydrique appliquée aux arbres de l'essai ont été expérimentés dans un dispositif en blocs randomisés à quatre répétitions. Chacun de ces traitements a porté sur huit arbres suivis pendant trois années consécutives, à raison de deux arbres par parcelle élémentaire. Les traitements ont consisté en des arbres non irrigués pendant toute la saison sèche (traitement 1 faisant office de contrôle) ; des

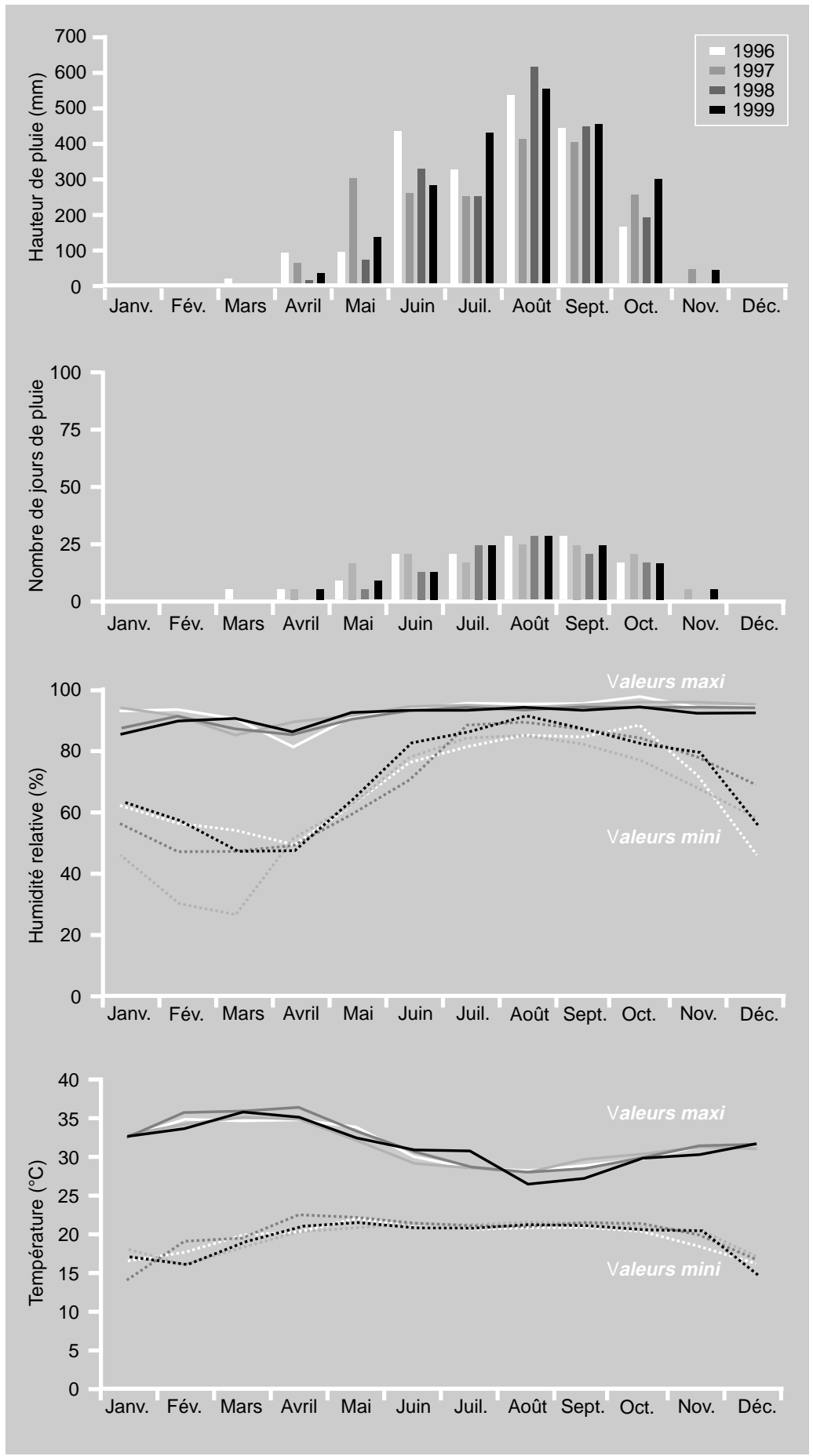

Figure 1.

Données agro-météorologiques relevées de 1996 à 1999 sur la station de Foulaya en Guinée ( $10^{\circ} 3^{\prime}$ lat. N, $12^{\circ} 52^{\prime}$ long. O, altitude $\left.380 \mathrm{~m}\right)$. 
arbres irrigués tous les 7 j à la dose de $1000 \mathrm{~L} \cdot \mathrm{mois}$, ce qui est proche de la capacité au champ (traitement 2) ; des arbres laissés sans irrigation pendant une certaine période de la saison sèche puis irrigués après $30 \mathrm{j}$ (traitement 3), $45 \mathrm{j}$ (traitement 4), $60 \mathrm{j}$ (traitement 5) ou $75 \mathrm{j}$ (traitement 6).

Pour chacun de ces quatre derniers traitements, la période de sécheresse a été interrompue par une série d'arrosages poursuivis pendant $3 \mathrm{j}$ afin de mouiller le sol occupé par les racines sur une profondeur moyenne de $30 \mathrm{~cm}$. Puis, une irrigation a été apportée tous les 10 j.

\subsection{Caractéristiques mesurées}

Les caractéristiques étudiées ont porté sur le relevé des dates de début et de pleine floraison et sur des données de production de fruits.

La floraison a été évaluée en dénombrant le nombre de boutons floraux verts observés $10 \mathrm{j}$ après l'interruption de la période de sécheresse, sur quatre rameaux localisés chacun à un des quatre points cardinaux de l'arbre. La fin de la floraison a été estimée, environ (5 à 10) j après le début du décompte, lorsque plus de la moitié des boutons floraux observés sur chaque rameau identifié avait atteint le stade d'épanouissement.

La production a été évaluée en pesant la récolte des arbres de la parcelle élémentaire puis en cumulant, par campagne, les poids obtenus pour chaque traitement. Une extrapolation a été faite pour déterminer les rendements obtenus par ha et par année.

\subsection{Analyses statistiques}

Les données de production par campagne ont été soumises à une analyse de variance simple traitée avec le logiciel Genstat 5.1 développé par l'université de Reading, Royaume Uni.

Une approche économique de l'étude a ensuite été amorcée.

\section{Résultats}

\subsection{Conditions climatiques}

Les données agro-météorologiques enregistrées au cours de l'étude (figure 1) ont été favorables aussi bien pour la mise en place de l'essai que pour le développement des arbres.

Pendant toute la durée de l'étude, il n'y a pas eu de pluies exceptionnelles en pleine saison sèche, de février à mars. Chaque année, la saison sèche a été stricte et effective à partir du $1^{\mathrm{er}}$ novembre, sauf en 1997 où, durant la première quinzaine de ce même mois, il y a eu 47,2 mm de pluie en quatre jours. Cela a entraîné un léger décalage du démarrage de l'essai.

Entre novembre 1996 et août 1999, soit pendant toute la durée de l'essai, il est tombé $5305,30 \mathrm{~mm}$ d'eau répartis sur 296 j. La pluviométrie moyenne annuelle de $1768,40 \mathrm{~mm} \cdot \mathrm{an}^{-1} \mathrm{a}$, a priori, largement dépassé les besoins moyens de la lime Tahiti estimés de (1000 à 1500) $\mathrm{mm} \cdot \mathrm{ha}^{-1}$. $\mathrm{an}^{-1}$. Par rapport à ces besoins, l'excédent théorique de $(268,40$ à 768,40$)$ mm d'eau tombés pendant environ 4 mois de la saison pluvieuse n'ont pu cependant empêcher qu'un déficit hydrique précoce peu perceptible soit présent dans le verger dès le début de la saison sèche. Les (4 à 7) mois dits " de déficits pluviométriques " ont induit, chaque année, la mise au repos végétatif des arbres.

Les variations de l'humidité relative ont été suffisantes pour accentuer les effets cumulés de la sécheresse chez des arbres dont le flétrissement tendait à se pérenniser au fur et à mesure des conditions drastiques et de l'avancée de la saison sèche.

Une saison sèche précoce et un retour tardif des précipitations tels que ceux observés pour la saison 1998/1999 ont semblé être des facteurs amplificateurs de l'effet des traitements ; ils ont conduit à augmenter le nombre d'irrigations à apporter (tableau II). Toutefois, faute d'observations échelonnées sur une période plus longue, ces faits n'ont pu être confirmés. 
Tableau II.

Nombre d'irrigations reçues par traitement (= type d'irrigation) et par campagne de production au cours d'un essai poursuivi de 1996 à 1999 pour étudier l'influence de la durée de la période de sécheresse sur les rendements de Citrus aurantifolia Christm. Swing. (Foulaya, Guinée).

\begin{tabular}{|c|c|c|c|}
\hline \multirow[t]{2}{*}{ Type d'irrigation } & \multicolumn{3}{|c|}{ Nombre d'irrigations apporté } \\
\hline & 1996-1997 & 1997-1998 & 1998-1999 \\
\hline Sans irrigation durant toute la saison sèche & 0 & 0 & 0 \\
\hline Irrigation tous les $7 \mathrm{j}$ durant la saison sèche & 26 & 21 & 28 \\
\hline Irrigation à $30 \mathrm{j}$ après le début de la saison sèche ${ }^{1}$ & 15 & 12 & 17 \\
\hline Irrigation à $45 \mathrm{j}$ après le début de la saison sèche ${ }^{1}$ & 13 & 9 & 16 \\
\hline Irrigation à $60 \mathrm{j}$ après le début de la saison sèche ${ }^{1}$ & 12 & 8 & 15 \\
\hline Irrigation à 75 j après le début de la saison sèche ${ }^{1}$ & 11 & 7 & 13 \\
\hline
\end{tabular}

\subsection{Floraison}

L'expérimentation mise en place a permis de mettre en évidence que la durée de restriction hydrique avait une influence sur la floraison de la lime Tahiti.

Les arbres privés d'eau pendant (30 ou 45) j, puis irrigués, ont présenté les premières ébauches florales $10 \mathrm{j}$ après l'interruption de la période de sécheresse. L'épanouissement des fleurs est intervenu dans les trois premières semaines qui ont suivi la mise en eau du verger. Parmi les six traitements appliqués, ce sont ceux qui ont conduit à faire des irrigations (30 ou 45) j après le début de la période de sécheresse qui ont été les plus efficaces pour favoriser l'induction florale et l'épanouissement des boutons floraux (tableau III). Les traitements témoins de "saison sèche totale " et "irrigation fréquente durant toute la saison sèche ", bien que fournissant aux arbres des conditions hydriques nettement opposées, ont cependant produit un nombre de fleurs très proche. Durant toute la période de l'expérimentation, la floraison et la production des arbres témoins non irrigués ont été conditionnées, chaque année, par le retour effectif des pluies. Les arbres irrigués tous les 7 j ont donné des floraisons occasionnelles ; toutefois, après les chaleurs de mars-avril, ces limettiers ont eu une légère tendance à fleurir de façon plus groupée.

\section{Tableau III.}

Nombre moyen de fleurs épanouies sur vingt rameaux fructifères du secteur sud de la frondaison de Citrus aurantifolia Christm. Swing., au cours des dix jours suivant la mise en eau d'un verger soumis à divers régimes de privation d'eau pendant la saison sèche (Foulaya, Guinée).

$\begin{array}{lc}\text { Type d'irrigation } & \text { Nombre de fleurs épanouies } \\ \text { Sans irrigation durant toute la saison sèche } & 53 \\ \text { Irrigation tous les } 7 \text { j durant la saison sèche } & 69 \\ \text { Irrigation à } 30 \text { j après le début de la saison sèche } & 316 \\ \text { Irrigation à } 45 \text { j après le début de la saison sèche } & 285 \\ \text { Irrigation à } 60 \text { j après le début de la saison sèche } & 196 \\ \text { Irrigation à } 75 \text { j après le début de la saison sèche } & 124\end{array}$


En conditions naturelles, sans apport d'eau intentionnel, la floraison de la lime s'est révélée fortement dépendante du climat. L'écart entre le début et la fin de floraison des arbres soumis à cette restriction hydrique a oscillé entre (2 et 6) semaines.

Les arbres irrigués après (30 ou 45) j de sécheresse ont eu une réponse précoce (tableau $I V$ ), alors que les arbres irrigués plus tardivement, soit après (60 ou 75) j de sécheresse, ont redémarré plus lentement. Dans ces derniers cas, les rameaux florifères ont été très nombreux et la floraison intense et groupée, cependant, les taux de nouaison ont été faibles. Cette dernière observation pourrait être liée à une réhumectation du sol et de l'atmosphère environnante alors insuffisante.

D’une façon générale, les arbres soumis à des traitements impliquant un arrêt de la période de sécheresse par la mise en route d'irrigations en cours de saison sèche ont montré, au cours des trois campagnes d'expérimentation, une reprise accélérée de leur cycle de développement. Quelques semaines après la mise en eau, de nouvelles ramifications, ainsi que des ébauches foliaires et florales viables ont pu être observées et cela avec d'autant plus d'intensité que la période de restriction hydrique avait été courte. Les floraisons des arbres soumis à des périodes de sécheresse de (60 et 75) j ont souvent péri du fait d'un dessèchement résultant de la faible hygrométrie ambiante et de l'état d'insaturation hydrique du sol.

\subsection{Production}

Du fait des traitements appliqués, la production a été étalée de fin avril à août (tableau IV), soit sur environ 4 mois, avec, en moyenne, deux à trois récoltes mensuelles. En avril, ce sont les fruits correspondant à la petite floraison de septembre/octobre et ceux formés à la suite des irrigations à (30 et 45) j après le début de la sécheresse qui ont été récoltés. Les meilleures limes, pesant en moyenne de (80 à 120) g, ont été obtenues entre fin mai et juillet. La coloration verte des fruits, à laquelle se rattache l'appellation commerciale " citron vert ", autre nom de la lime, a été plus intense à partir de la fin du mois de juin.

Le chevauchement floraison/fructification observé du fait des traitements appliqués s'est répercuté sur la durée totale du temps de récolte réelle qui a duré près de 4 mois. Cela a permis d'élargir la période de récolte normale et d'obtenir une production de fruits sur ( 8 à 9) mois, environ.

L'influence de la durée des périodes de sécheresse imposées a été perceptible aussi bien à l'échéance d'une même année qu'à celle de plusieurs campagnes. Les effets mis en évidence au cours de notre étude ont donc été validés à la fois par les traitements appliqués et par la durée d'expérimentation.

Le traitement impliquant une irrigation continue pendant toute la saison sèche a

\section{Tableau IV. \\ Dates moyennes de floraison et de récolte pour des limettiers Tahiti soumis à divers régimes de restriction hydrique pendant les saisons sèches des années 1996 à 1999 à Foulaya, Guinée.}

\begin{tabular}{|c|c|c|c|c|}
\hline \multirow[t]{2}{*}{ Type d'irrigation } & \multicolumn{2}{|c|}{ Date de floraison } & \multicolumn{2}{|c|}{ Date de récolte } \\
\hline & Début & Fin & Début & Fin \\
\hline Sans irrigation durant toute la saison sèche & Fin avril à mai & mai à juin & Septembre à octobre & Janvier \\
\hline Irrigation tous les 7 j durant la saison sèche & Imprécis & Imprécis & Imprécis & Imprécis \\
\hline Irrigation à 30 j après le début de la saison sèche & 10 décembre & 25 au 30 décembre & Fin avril & Mai à juin \\
\hline Irrigation à 45 j après le début de la saison sèche & 25 au 30 décembre & 15 janvier & Fin mai & Juin \\
\hline Irrigation à 60 j après le début de la saison sèche & 15 au 20 janvier & 5 au 10 février & Juin & Juillet à août \\
\hline Irrigation à 75 j après le début de la saison sèche & 1 au 5 février & 20 au 25 février & Fin juin & Août \\
\hline
\end{tabular}


Tableau V.

Production de limettiers Tahiti de contre-saison soumis à divers régimes de restriction hydrique pendant les saisons sèches des années 1996 à 1999 à Foulaya, Guinée.

\begin{tabular}{|c|c|c|c|}
\hline \multirow[t]{2}{*}{ Type d'irrigation } & $\begin{array}{c}\text { Production } \\
\text { (kg de fruits pour deux arbres par répétition) }\end{array}$ & \multirow[t]{2}{*}{$\begin{array}{l}\text { Production } \\
\text { moyenne }^{1}\end{array}$} & \multirow{2}{*}{$\begin{array}{l}\text { Classement } \\
\text { des } \\
\text { traitements }\end{array}$} \\
\hline & Répétition 1 Répétition 2 Répétition 3 Répétition 4 & & \\
\hline
\end{tabular}

(a) Campagne 1996 / 1997

Sans irrigation durant toute la saison sèche Irrigation tous les $7 \mathrm{j}$ durant la saison sèche

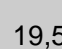
Irrigation à $30 \mathrm{j}$ après le début de la saison sèche Irrigation à $45 \mathrm{j}$ après le début de la saison sèche Irrigation à $60 \mathrm{j}$ après le début de la saison sèche Irrigation à $75 \mathrm{j}$ après le début de la saison sèche

$\begin{array}{llllll}19,5 & 27,0 & 21,0 & 24,5 & 23,0 & \mathrm{e} \\ 24,8 & 23,0 & 27,5 & 24,3 & 24,9 & \mathrm{e} \\ 76,5 & 85,0 & 80,3 & 78,7 & 80,1 & \mathrm{a} \\ 65,0 & 70,2 & 64,3 & 57,7 & 64,3 & \mathrm{~b} \\ 40,5 & 37,5 & 43,8 & 47,4 & 42,3 & \mathrm{~cd} \\ 40,2 & 35,3 & 45,5 & 38,7 & 39,9 & \mathrm{~d}\end{array}$

${ }^{1}$ Erreur standard : $2,04 \mathrm{~kg}$; cœfficient de variation : $9 \%$.

\section{(b) Campagne 1997 / 1998}

Sans irrigation durant toute la saison sèche Irrigation tous les $7 \mathrm{j}$ durant la saison sèche Irrigation à $30 \mathrm{j}$ après le début de la saison sèche Irrigation à $45 \mathrm{j}$ après le début de la saison sèche Irrigation à $60 \mathrm{j}$ après le début de la saison sèche Irrigation à $75 \mathrm{j}$ après le début de la saison sèche

$\begin{array}{rrrcrc}59,3 & 70,5 & 65,7 & 63,8 & 64,8 & \mathrm{c} \\ 44,5 & 53,4 & 40,2 & 48,2 & 46,6 & \mathrm{~d} \\ 105,2 & 90,3 & 95,8 & 115 & 101,6 & \mathrm{a} \\ 89,2 & 108,0 & 90,3 & 96,7 & 96,1 & \mathrm{ab} \\ 83,0 & 74,5 & 92,7 & 98,3 & 87,1 & \mathrm{~b} \\ 75,5 & 86,4 & 90,3 & 92,2 & 86,1 & \mathrm{~b}\end{array}$

${ }^{1}$ Erreur standard : $4 \mathrm{~kg}$; cœefficient de variation : $10 \%$.

\section{(c) Campagne $1998 / 1999$}

Sans irrigation durant toute la saison sèche Irrigation tous les $7 \mathrm{j}$ durant la saison sèche Irrigation à $30 \mathrm{j}$ après le début de la saison sèche Irrigation à $45 \mathrm{j}$ après le début de la saison sèche

$\begin{array}{rccrc}84,3 & 81,3 & 70,6 & 73,2 & 77,4 \\ 74,8 & 84,7 & 57,5 & 55,1 & 68 \\ 142,8 & 126,5 & 123,8 & 151,7 & 136 \\ 122,3 & 151 & 109,1 & 117,8 & 125,1 \\ 92,9 & 82,7 & 107 & 108,1 & 97,7 \\ 83,3 & 119,9 & 111 & 96,5 & 102,5\end{array}$

$c$
$c$
$a$
$a$
$b$
$b$

${ }^{1}$ Erreur standard : 7,5 kg ; cœfficient de variation : 14,8\%.

eu un effet dépressif sur la production, quelle que soit la campagne considérée (tableau $V$ ) ; son rendement de 7,6 $\mathrm{t} \cdot \mathrm{ha}^{-1} \mathrm{a}$ été significativement le moins élevé de tous les autres traitements expérimentés (tableau $V T$ ).

Les traitements faisant intervenir la mise en route d'irrigations (30 et 45) $\mathrm{j}$ après le début de la saison sèche ont conduit aux rendements moyens les plus élevés de (16,8 et 15,1$) \mathrm{t} \cdot \mathrm{ha}^{-1}$ (tableau VI).

\subsection{Approche économique de l'étude}

À partir du nombre moyen d'irrigations reçues, du rendement moyen obtenu par traitement au cours des trois années d'expérimentation, et du prix de vente "bordchamp " de $150 \mathrm{FG} \cdot \mathrm{kg}^{-1}$, les revenus bruts moyens ont pu être estimés (tableau VI).

La rentabilité de la technique de production de limes de contre-saison par mâ̂trise des dates d'irrigation décalées s'est 
Tableau VI.

Estimation des revenus bruts moyens en francs guinéens ${ }^{1}(\mathrm{FG})$, sur la base d'un prix de vente de $150 \mathrm{FG} \cdot \mathrm{kg}^{-1}$, pour des limes Tahiti de contre-saison dont les arbres ont été soumis à divers régimes de privation d'eau pendant les saisons sèches des années 1996 à 1999 à Foulaya, Guinée.

\begin{tabular}{lccc} 
Type d'irrigation & $\begin{array}{c}\text { Rendement } \\
\left(\mathrm{t} \cdot \mathrm{ha} \mathrm{a}^{-1}\right)\end{array}$ & $\begin{array}{c}\text { \% accroissement } \\
\text { par rapport au témoin }\end{array}$ & $\begin{array}{c}\text { Revenus bruts } \\
\left(10^{3} \mathrm{FG} \cdot \mathrm{ha}^{-1}\right)\end{array}$ \\
\hline Sans irrigation durant toute la saison sèche & 8,7 & 100 & 1305 \\
Irrigation tous les 7 j durant la saison sèche & 7,6 & $-12,6$ & 1140 \\
Irrigation à 30 j après le début de la saison sèche & 16,8 & 93,0 & 2520 \\
Irrigation à 45 j après le début de la saison sèche & 15,1 & 73,6 & 2265 \\
Irrigation à 60 j après le début de la saison sèche & 12,0 & 37,9 & 1800 \\
Irrigation à 75 j après le début de la saison sèche & 12,4 & 42,5 & 1860 \\
1 250 francs guinéens $=6,55$ euros. & & &
\end{tabular}

révélée avantageuse : les traitements impliquant des périodes de sécheresse de (30, 45,60 et 75) j ont été à la fois plus productifs et plus rentables que les deux témoins sans arrosage du tout ou avec irrigation continue. De plus, cette dernière technique est devenue de plus en plus chère avec le temps et, par conséquent, moins rentable.

Les traitements permettant d'interrompre la période de sécheresse (30 ou 45) j après le début de la saison sèche, entrant en production alors que les réserves en eau du sol étaient encore accessibles, ont permis de mieux valoriser l'irrigation des arbres. L'accroissement de production alors obtenu a été le double de celui des traitements impliquant une irrigation après (60 ou 75) j de sécheresse.

Par ailleurs, la production de contre-saison déclenchée et entretenue en saison sèche a permis de minimiser les pertes qui auraient pu être occasionnées par l'anthracnose apicale, fréquente en août-octobre, du fait même que les récoltes ont pu avoir lieu avant la pleine prolifération des maladies fongiques redoutées à partir de juillet.

\section{Discussion}

L'absence de pluies exceptionnelles horssaison pendant toute la période de l'expé- rimentation a permis d'éviter la surestimation des taux de floraison/nouaison et des rendements extrapolés qui auraient pu être faussés par de telles précipitations.

Chez les traitements témoins (aucune irrigation et irrigation continue), des à-coups de floraison et de production ont pu être constatés ; ils sont généralement à attribuer au réveil tardif de certains bourgeons floraux. Dans le cas de l'absence totale d'irrigation, ces floraisons accidentelles pourraient être expliquées par la proximité d'arbres ayant subi l'un des traitements avec arrêt contrôlé de la période de sécheresse. Ce pourrait être une carence du dispositif expérimental utilisé que les précédents chercheurs n'avaient pas ressorti. Globalement, les résultats obtenus au cours de nos travaux confirment ceux d'autres auteurs qui ont travaillé sur des périodes de ( 4,8 et 12) semaines $[3,57,10]$.

L'induction florale, intervenant entre (10 et 15) j après la mise en eau, n'a pas été très différente des (20 à 28) j rapportés par d'autres auteurs et qui correspondraient à une moyenne générale obtenue dans le cas des principaux agrumes commerciaux $[3,10]$. Par ailleurs, l'intervalle de (15 à 45) j entre le début et la fin de floraison est resté sensiblement analogue à celui signalé auparavant dans d'autres travaux relatifs sur agrumes et autres fruitiers [3, 10, 12, 13]. 
La densité des fleurs et la floraison groupée, observées au sein de la canopée des limettiers irrigués après (60 ou 75) j de sécheresse, ont été d'autant plus élevées que les arbres avaient été éprouvés par la restriction hydrique. L'effet de cette privation a été accentué par la faible hygrométrie ambiante de cette époque et il s'en est suivi une chute de fruits néoformés et en croissance. Cependant, il n'existe pas, a priori, de relation directe entre la densité de floraison et la réussite de la nouaison qui serait fortement dépendante des régimes hydriques et nutritionnels apportés aux arbres en cette étape critique. Un stress hydrique long et intense, même s'il conduit à une forte floraison des arbres, ne constituerait donc qu'un préalable à la production qu'il ne suffirait pas à déterminer de façon unilatérale.

Les moyennes de production obtenues au cours de cet essai sur limettiers ne se sont pas écartées des moyennes habituelles observées pour cette variété conduite en verger intensif. Cependant, les fruits ont été homogènes, brillants et teintés d'un verttournant attractif. Leur entrée opportune, en contre-saison, dans un marché demandeur, a créé une forte plus-value, de l'ordre de 1 à 6 en moyenne, qui correspond à une augmentation du prix de vente de (150 à 800) $\mathrm{FG} \cdot \mathrm{kg}^{-1}$. La qualité des fruits et l'époque de leur mise sur le marché constituent donc un avantage certain pour ces limes par rapport à celles produites sans irrigation.

Au cours de notre expérimentation, une durée de restriction hydrique inférieure à 2 mois a eu une incidence positive sur la viabilité de la floraison, de la nouaison et de la production. Ce résultat a déjà été souligné sur diverses espèces fruitières [3, 12-15] alors que d'autres travaux ont mis en évidence qu'une telle irrigation non suivie d'apports d'eau suffisants ou régulièrement assurés pouvait présenter un sérieux handicap pour ces mêmes paramètres de floraison, nouaison et production [10, 12, 14, 19].

La récolte obtenue sur les arbres n'ayant subi que (30 ou 45) j de sécheresse a été plus échelonnée que celle des limettiers des autres traitements qui ont eu tendance à présenter un groupage de la maturité des fruits et donc de la récolte. Par ailleurs, l'intérêt d'apporter l'eau même tardivement (mais pas trop tardivement) s'est répercuté sur la production : les rendements obtenus avec les modalités de (60 ou 75) j de sécheresse ont été supérieurs à celui obtenu lors du traitement impliquant une irrigation continue. Ce constat confirme, à quelques exceptions près, l'hypothèse selon laquelle les agrumes ont besoin de subir les effets d'une petite sécheresse (repos végétatif) pour produire rentablement et durablement [2-4, 19].

Globalement, quel qu'ait été le traitement appliqué, la commercialisation des limes vendues soit en petites quantités mais en plusieurs offres, soit en plus grand volume du fait des récoltes groupées a présenté des avantages certains qui n'avaient pas été mis en évidence lors de précédentes études sur ce thème.

À partir des résultats obtenus, une nouvelle approche dans la gestion de l'eau à apporter, en saison sèche, en vergers de limettiers, pourrait être proposée afin d'obtenir un étalement de la floraison et de la production pour un rendement optimal du verger. Elle consisterait à instaurer, sur différents secteurs de la parcelle, trois périodes de rationnement en eau de ( 4 à 5) semaines chacune, décalées dans le temps sur une durée de 15 semaines, chaque secteur devant être à sec lors de l'une des trois périodes.

\section{Conclusions}

Au terme des trois années d'expérimentation, le décalage de la production de limes par la maîtrise de la durée de restriction hydrique est apparu être une activité rentable pour la mise en marché de limes Tahiti de contre-saison. Dans ce contexte, des durées de sécheresse de (30 et 45) j se sont révélées être les plus favorables dans les conditions de Foulaya en Guinée. Même avec un système d'irrigation peu fiable (irrigation à la raie), la marge bénéficiaire estimée pourrait alors être doublée. 
Bien que nos travaux aient confirmé qu'une période de restriction hydrique était absolument nécessaire pour favoriser l'entrée des arbres en floraison, ils ont également souligné le rôle préjudiciable de 6 mois de sécheresse susceptibles de conduire les limettiers à un état proche du flétrissement permanent.

L'approche pour la gestion de l'apport d'eau telle que celle proposée à l'issue de ces travaux pourrait trouver une efficacité technique et pratique ainsi qu'une justification économique dans les plantations industrielles de limettiers ou d'autres agrumes, où l'irrigation peut être maîtrisée.

La validation de cette technique de production de contre-saison reste à faire, en particulier dans les zones agrumicoles de la Guinée où la cercosporiose des agrumes (Phaeoramularia angolensis) est en train de sévir.

\section{Références}

[1] Cassin J., Haendler L., Notes sur les caractères commerciaux de quelques variétés d'agrumes cultivées en Guinée, Fruits 9 (10) (1954) 452-455.

[2] Cassin J., influence du climat sur la floraison des citrus en Guinée, Fruits 13 (7) (1958) 286-292.

[3] Southwick S.M., Davenport T.L., Characterization of water stress and low temperature effects on flower induction in citrus, Plant Physiol. 81 (1986) 26-29.

[4] Anonyme, institut de recherches sur les fruits et agrumes. Une expérience de 40 années, Fruits hors-série, tome 2, IRFA, Paris, 1987, p. 16.

[5] Cassin J., Bourdeaut J., Fouqué A., Furon V., Gaillard J.P., Lebourdelles J., Montagut G., Moreuil C., The influence of climate upon the blooming of citrus in tropical areas, in: 1st Int. Citrus Symp., Vol. 1, Riverside, USA, 1969, 315-323.
[6] Davenport T.L., Citrus flowering, Hortic. Rev. 12 (1960) 349-408.

[7] Pire R., Rojas E., Effect of drought stress and urea sprays on production of flower and vegetative buds of lime Tahiti, Fruits 54 (3) (1999) 177-182.

[8] Fouqué A., Essai irrigation agrumes en Côte d'Ivoire, Fruits 35 (3) (1960) 159-164.

[9] Fouqué A., Combres J.C., Études sur l'irrigation en Côte d'Ivoire, Fruits 33 (12) (1978) 845-847.

[10] Cassin J., Le Bourdelles J., Médori J.P., L'irrigation des agrumes en Corse. Techniques et nouveaux systèmes, Bull. Somivac $n^{\circ} 91$, Paris, France, 1973, 63-70.

[11] Li S.H., Huguet J.C., Schock P.G., Bussi C., Réponses de jeunes pêchers cultivés en pots à différents régimes d'alimentation hydrique. II : effets sur la croissance et le développement, Agronomie 10 (1990) 353-360.

[12] Goguey T., Approche architecturale des mécanismes de la croissance aérienne et de la floraison du manguier, thèse, Univ. Montpellier II, Montpellier, France, 1995.

[13] Sahli A., Grab M., Ben Mechila N., Méthodologie pour étudier l'effet de l'alimentation hydrique sur la croissance du pêcher, Fruits 54 (3) (1999) 197-206.

[14] Chalmers D.J., Mitchell P.D., Van Heek L., Control of peach tree growth and productivity by regulated water supply, tree density, and summer pruning, J. Am. Soc. Hortic. Sci. 106 (3) (1981) 307-312.

[15] Anonyme, L'Irag (Institut de recherche agronomique de Guinée) : bilan et perspective de I'Irag en 1996, Cirad, Montpellier, France, 1997, p. 28.

[16] Louguet P., Laffray D., Techniques d'études de l'état hydrique des plantes, Bull. Amélior. Prod. Agric. Milieu Arid. 1 (1988) 7-34.

[17] Isbérie C., L'eau du sol : principes généraux, moyens de mesure, Arboricult. Fruit. 34 (396) (1987) 39-44.

[18] Poss R., Quelques voies actuelles de la dynamique de l'eau dans le sol en zone inter tropicale, Fruits 45 (5) (1990) 507-510.

[19] Xiloyannis C., Uriu K., Martin G.C., Seasonal and diurnal variation in abscissic acid, water potential, and diffusive resistance in leaves from irrigated and non irrigated peach trees, J. Am. Soc. Hortic. Sci. 105 (3) (1980) 412-415. 


\section{Influencia de la duración de la restricción hídrica en la producción de la lima Tahití (Citrus aurantifolia Christm. Swing.) en Guinea.}

Resumen - Introducción. En Guinea, el cultivo de los cítricos está sujeto al comienzo de la estación de lluvias. Por esta razón, su producción se agrupa de octubre a enero y están prácticamente ausentes de los mercados el resto del año. Se emprendió una iniciativa destinada a escalonar la producción de estos árboles, y especialmente la del limero Tahití, a partir de un mejor control de la duración del período de sequía. Material y métodos. El estudio efectuado de 1996 a 1999 en la estación de Foulaya (Guinea) permitió comparar seis tratamientos compuestos por un tratamiento de árboles con riego continuo y cinco tratamientos con duraciones de restricción hídrica variable [estrés hídrico total o sequía de $(30,45,60$ y 75) d. El diseño comprendía cuatro bloques aleatorios y la parcela elemental dos árboles por tratamiento. Se estudiaron, durante cuatro campañas de cosecha, la floración y producción de los árboles tratados. Resultados y discusión. Se obtuvieron diferentes respuestas en cuanto a la reanudación del crecimiento, fecha, densidad y duración de la floración y la producción. El riego continuo tuvo un efecto depresivo en el rendimiento de $-12,6 \%$. La producción se prolongó de ( 4 a 5) meses suplementarios (de finales de abril a agosto) permitiendo una mayor presencia de la lima Tahití en el mercado. La plusvalía de la producción de fuera de temporada fue 1,5 a 2,5 veces superior a la de temporada. Conclusión. Los períodos de sequía limitados a (30 ó 45) d mediante control de riego permitieron escalonar y obtener rendimientos anuales de $(16,8$ y 15,1$)$ t $\cdot \mathrm{ha}^{-1}$ de limas económicamente más rentables. Esta técnica se podría emplear con provecho en plantaciones comerciales de limas Tahití de fuera de temporada.

Guinea / Citrus aurantifolia / estrés de sequia / productos fuera de temporada / floración / rendimiento / calendario de los riegos

To access this journal online: www.edpsciences.org 